\title{
Are Diabetic Older Adults Equally Satisfied with Remote Consultation vs. Face to Face Consultation? \\ Shelby Lanie ${ }^{1}$, Erin Jefferson ${ }^{2}$, Brian Henriksen ${ }^{2}$ \\ ${ }^{1}$ Indiana University School of Medicine; ${ }^{2}$ Fort Wayne Medical Education Center
}

\section{Background and Hypothesis:}

The COVID-19 pandemic has likely made remote consultation (RC) a permanent part of healthcare. Older adults could greatly benefit from RC, and current literature indicates older adults are equally satisfied with RC vs. face to face consultation (FTFC). However, there is selection bias in the literature towards adults who feel comfortable enrolling in a technology study. We hope to fill this dearth by surveying diabetic older adults who were strongly encouraged by their physicians to utilize RC during the COVID-19 quarantine. We hypothesize older adults will be less satisfied by RC vs. FTFC.

\section{Experimental Design or Project Methods:}

We propose a retrospective case control cross-sectional telephone survey (data collected in RedCap) examining satisfaction with consultation in diabetic older adults who received care via $\mathrm{RC}$ during the spring 2020 COVID-19 quarantine. We will compare the RC condition to the FTFC sample (appointments that occurred before quarantine) using an unpaired t test. We also propose measuring commute time/distance and hearing difficulty as possible mediating factors on satisfaction, analyzed with a regression analysis and a chi square analysis respectively

\section{Results:}

We expect to see lower satisfaction rates in the RC vs. the FTFC condition. However, we expect this effect to be mediated by patients' typical commute time/distance to their clinic. We also expect to see lower rates of satisfaction in the RC condition than the FTFC condition for patients with hearing difficulty.

\section{Conclusion and Potential Impact:}

The population is continuing to age, suffering from increased rates of diabetes and other chronic conditions that require more frequent, and more burdensome appointments. While RC has incredible potential to help older adults keep important appointments, their satisfaction is an integral part of successful care. This study could help dictate clinical decisions by informing providers and patients on older adults' satisfaction with RC. 